\title{
Dynamics of fluctuations in an optical analogue of the Laval nozzle
}

\author{
Itzhak Fouxon, ${ }^{1}$ Oleg Farberovich, ${ }^{1}$ Shimshon Bar-Ad, ${ }^{1}$ and Victor Fleurov ${ }^{1}$ \\ ${ }^{1}$ Raymond and Beverly Sackler School of Physics and Astronomy, Tel-Aviv University, Tel-Aviv 69978, Israel
}

(Dated: March 15, 2022)

\begin{abstract}
Using the analogy between the description of coherent light propagation in a medium with Kerr nonlinearity by means of nonlinear Schrödinger equation and that of a dissipationless liquid, we propose an optical analogue of the Laval nozzle. The optical Laval nozzle will allow one to form a transonic flow, in which one can observe and study very unusual dynamics of classical and quantum fluctuations, including an analogue of the Hawking radiation of real black holes. Theoretical analysis of these dynamics is supported by numerical calculations, and estimates for a possible experimental realization are presented.
\end{abstract}

PACS numbers: 42.65.Sf; 47.40.Hg

Black hole radiation is one of the most impressive phenomena at the intersection of general relativity and quantum field theory. Accounting for the quantum nature of the physical vacuum led to the prediction that a black hole, defined classically as an object that even light cannot escape, in fact can be characterized by a temperature, entropy [1] and moreover emits thermal radiation [2]. Since direct experimentation with black holes is hardly possible, it was suggested to consider analogous phenomena in condensed matter physics where the "high-energy" (short-wavelength) physics is known [3]. The suggestion was based on the observation that the derivation of the Hawking radiation uses only the linear wave equation in curved space-time, and not the Einstein equations. The same conditions for wave propagation arise when considering sound propagation in a fluid when the background flow is non-trivial 3]. This similarity was shown to be sufficient for applying the original considerations by Hawking, predicting the existence of thermal radiation of quantum origin from the fluid counterpart of the horizon, which can be called Mach horizon (where the fluid velocity equals the sound velocity, i.e. Mach number $M=1)$. These ideas were further developed for possible applications in BEC fluids and other systems [4 -8]. As for experimental realizations, a white hole horizon was observed in optical fibers [9], where the probe light was back-reflected from a moving soliton, and a black hole horizon was observed in a BEC system [10].

We propose an experimental setup capable of creating a Mach horizon in an optical medium with Kerr nonlinearity, which may be called optical Laval nozzle. The propagation of weakly nonlinear coherent optical pulses is described by the non-linear Schrödinger (NLS) equation

$$
i \frac{\partial A}{\partial z}=-\frac{1}{2 \beta_{0}} \nabla^{2} A+U(x, y) A+\lambda|A|^{2} A .
$$

It assumes the paraxial approximation, according to which the electric field of the light wave is written as $E=A(x, y, t ; z) e^{-i \beta_{0} z}$, where $A(x, y, t ; z)$ is weakly $z$ dependent complex amplitude of the light propagating in the $z$ direction. The time coordinate $t$ is converted into the $\tau$ coordinate 11 which describes the shape of the light pulse in the moving coordinate system. The radius vector differential is now $d \boldsymbol{r}=(d x, d y, d \tau)$ (anomalous dispersion) or $d \boldsymbol{r}=(d x, d y,-i d \tau)$ (normal dispersion). $U(x, y)$ is the equivalent external potential created by spacial variation of the refraction index in the medium and does not depend on $\tau$.

Now we briefly sketch the fundamental result [3] as applied to coherent light propagation in a medium with Kerr nonlinearity. The Madelung transformation 12 $A=f e^{i \phi}$ (see also 13 15]) maps the NLS eq.(1) on two equations

$$
\begin{aligned}
& \partial_{z} \rho+\nabla \cdot[\rho \boldsymbol{v}]=0, \\
& \partial_{z} \boldsymbol{v}+\frac{1}{2} \nabla \boldsymbol{v}^{2}=-\frac{1}{\beta_{0}} \nabla\left(V_{q u}+U+\lambda \rho\right)
\end{aligned}
$$

for an equivalent fluid with density $\rho=f^{2}$ and velocity $\beta_{0} \boldsymbol{v}=-\nabla \phi$. For permanently shined light the coordinate $\tau$ is redundant. $V_{q u}=-\frac{1}{2 \beta_{0}} \frac{\nabla^{2} f}{f}$ is a "quantum potential" corresponding to $\hbar=1$. The light wave vector $\beta_{0}$ is the "particle mass" and the velocity $v$ is dimensionless.

Linearizing Eqs. (2) and (3) with respect to the small fluctuations $\rho-\rho_{0}=\rho_{0} \psi$ and $\phi-\phi_{0}=\varphi$ around a steady solution $\rho_{0}(x, y)$ and $\varphi_{0}(x, y)$, we arrive at

$$
(-g)^{-1 / 2} \partial_{\mu}(-g)^{1 / 2} g^{\mu \nu} \partial_{\nu} \varphi=0,
$$

where the metric $g^{\mu \nu}$ with the determinant $g$ is given by the interval

$$
d \sigma^{2}=g_{\mu \nu} d x^{\mu} d x^{\nu}=
$$

$$
\sqrt{\frac{\beta_{0}}{\lambda \rho_{0}}}\left[d \boldsymbol{r}^{2}-2 d z \boldsymbol{v}_{0} \cdot d \boldsymbol{r}-\left(\frac{\lambda \rho_{0}}{\beta_{0}}-v_{0}^{2}\right) d z^{2}\right]
$$

with $g^{\mu \mu^{\prime}} g_{\mu^{\prime} \nu}=\delta_{\nu}^{\mu}$. The ordinary "sound waves" in the effective fluid arise as solutions of eq. (4) around the equilibrium solution $\rho_{0}=$ const, $\phi_{0}=$ const and $\boldsymbol{v}_{0}=0$, that 
exists at $U=0$, so that $\lambda \rho_{0} / \beta_{0}=s^{2}$ is the squared sound velocity. The nonlinearity coefficient is positive, $\lambda>0$, otherwise the "sound velocity" becomes imaginary and various instabilities, such as collapsing solitons [16] arise. Eq. (44) may include corrections due to the quantum potential, which may be of importance for short waves.

The background flow may arise in the Laval nozzle 17, 18], i.e. a vessel with variable cross-section $S(x)$, whose application to some condensed-matter analogues was discussed in [19, 20]. The flow velocity increases with decreasing $S(x)$, and reaches the sound velocity in the narrowest part of the vessel, called the throat. Further acceleration is reached by increasing $S(x)$. Here we analyze the transonic flow for the optical realization of the Laval nozzle shown in Fig. ??. The laser beam, nearly

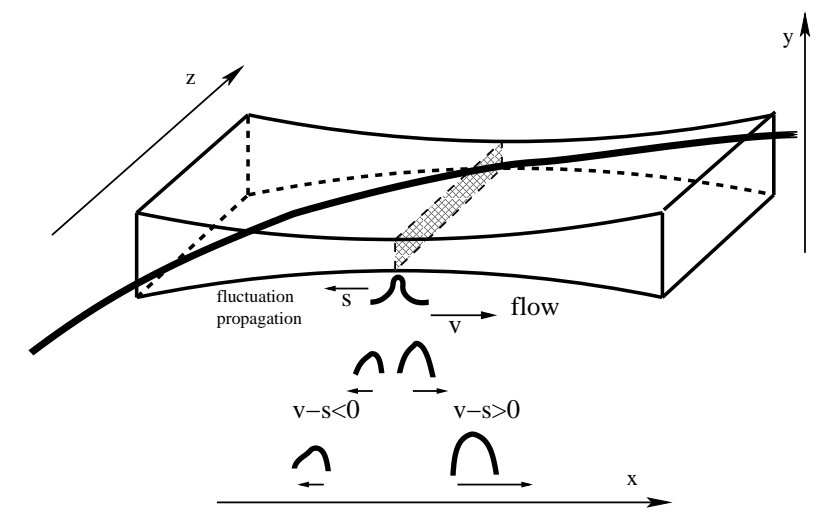

FIG. 1: A cartoon of a laser beam propagating inside the optical Laval nozzle. The beam, with a small initial angle relative to the $\mathrm{z}$ axis, bends more towards the $\mathrm{x}$ direction as it propagates along the z direction ("time"). The bend in the cartoon is strongly exaggerated. A fluctuation is schematically shown near the Mach horizon as it is cut in two parts which propagate in opposite directions.

parallel to the $z$ axis, is tilted in the $x$ direction by an angle $\arctan \left(k_{x} / \beta_{0}\right)$, i.e. the initial phase in the electric field amplitude $A(x, y)$ is $\varphi=k_{x} x . k_{x} / \beta_{0}$ plays the part of "flow velocity" in the $x$ direction. Acceleration of the flow within the plate of variable width ( $y$ - axis) results in bending of the beam ( $k_{x}$ increases) as shown in Fig. ??. It leads to small corrections to $\beta_{0}$, quadratic in $k_{x} / \beta_{0} \ll 1$. We always assume that the wave length $1 / \beta_{0}$ is smaller than all the other relevant scales.

Now we consider the flow bounded by the walls of a hyperbolic shape

$$
\frac{y^{2}}{a^{2}}-\frac{x^{2}}{b^{2}}=1
$$

with sliding boundary conditions, and the elliptic coordinates $x=c \sinh u_{1} \sin u_{2}, \quad y=-c \cosh u_{1} \cos u_{2}$, are introduced, with $c=\sqrt{a^{2}+b^{2}}$. From the symmetry considerations the flow is along the $\mathrm{x}$ axis for $y=0$, i.e. $u_{2}=\pi / 2$.
Equation (2) for a steady flow becomes

$$
\frac{\partial}{\partial u_{1}} \sqrt{\Delta}\left(\rho v_{1}\right)+\frac{\partial}{\partial u_{2}} \sqrt{\Delta}\left(\rho v_{2}\right)=0
$$

where $\Delta=\sinh ^{2} u_{1}+\sin ^{2} u_{2}$ and $v_{1}$ and $v_{2}$ are the velocity components in the elliptic coordinates.

This equation is satisfied if

$$
\begin{aligned}
& \rho v_{1}=\frac{F_{1}\left(u_{2}\right)+f u_{1}}{\sqrt{\Delta}}, \\
& \rho v_{2}=\frac{F_{2}\left(u_{1}\right)-f u_{2}}{\sqrt{\Delta}}
\end{aligned}
$$

where $F_{1}\left(u_{2}\right)$ and $F_{2}\left(u_{1}\right)$ are functions only of $u_{2}$ and $u_{1}$ respectively, and $f$ is a constant. Since the transversal velocity $v_{2}$ is zero at the throat at $u_{1}=0$ we get that $f=0$.

We assume the streamlines in the vicinity of the central line, i.e. small $\widetilde{u}_{2}=u_{2}-\frac{\pi}{2}$, to be close to the lines of constant $\widetilde{u}_{2}$. Then calculating the covariant derivatives in the curvilinear elliptic coordinates and neglecting in the result the second order corrections in $\widetilde{u}_{2}$, Eq. (3) reads (cf., [18])

$$
\frac{\partial\left(\rho v_{1}\right)}{\partial u_{1}}=\rho\left(1-\frac{v_{1}^{2} \beta_{0}}{\lambda \rho}\right) \frac{\partial v_{1}}{\partial u_{1}} .
$$

The flow reaches the sound velocity at $u_{1}=0$. The second Eq. (7) in the elliptic coordinates results in $\rho\left(u_{1}\right) v_{1}\left(u_{1}\right)=d-\frac{1}{2} d u_{1}^{2}$ with $d=F_{1}(\pi / 2)=\bar{\rho} \bar{s}$, and finally, using Eq. (8), we get $v_{1}=\bar{s}\left(1+\frac{u_{1}}{\sqrt{3}}\right), \quad \rho=$ $\bar{\rho}\left(1-\frac{u_{1}}{\sqrt{3}}\right)$, where overlined quantities refer to the throat. The coordinate dependence of the density immediately yields the coordinate dependence of the sound velocity $\lambda \rho\left(u_{1}\right)=\beta_{0} s^{2}\left(u_{1}\right)$. Correspondingly the values of these quantities at the throat are connected as $\lambda \bar{\rho}=\beta_{0} \bar{s}^{2}$.

We may now represent Eq. (4) in the narrow transonic region in the dimensionless form

$$
\partial_{\zeta}^{2} \varphi+2 \partial_{\xi} \partial_{\zeta} \varphi+\alpha \xi \partial_{\xi}^{2} \varphi+\alpha_{1} \partial_{\zeta} \varphi+\alpha \partial_{\xi} \varphi=0,
$$

where $\zeta=\frac{\bar{s}}{c} z, \quad \xi=u_{1} \approx \frac{x}{c}$. The coefficients $\alpha_{1}=$ $1 / \sqrt{3}$, and $\alpha=\sqrt{3}$ are obtained above for the hyperbolic Laval nozzle. However, the ratio $\alpha / \alpha_{1}=3$ holds for any differentiable shape of this type. We look for a solution of Eq. (??) in the form $\varphi(\xi, \zeta)=\exp \{i \nu \zeta\} \bar{\varphi}(\xi)$, where the functions $\bar{\varphi}(\xi)$ are linear combinations of the two sets of eigen functions

$$
\bar{\varphi}_{1}(\xi)=e^{\frac{-i \nu}{\alpha} \ln (-\xi)} \text { and } \bar{\varphi}_{2}(\xi)=e^{-i k(\nu) \xi} .
$$

In a background flow characterized by a space independent velocity, eq. (9) has the form of a one-dimensional Klein-Gordon wave equation for the field $\varphi$ in a flat space. Then one obtains two plane waves propagating to the left 
(against the flow) and to the right (with the flow). In the considered here transonic case, when the background flow velocity and speed of sound vary with the coordinate, the left moving plane wave transforms into the eigen mode $\bar{\varphi}_{1}(\xi)$, which is a function with a branching point near $\xi=0$, whereas the right moving plane wave is characterized by the nonlinear spectral relation

$$
k(\nu)=\frac{\nu}{2} \frac{1-i /(\nu \sqrt{3})}{1-i \sqrt{3} /(2 \nu)} .
$$

The complex wave vector for the real "frequencies" reflects the fact that the coordinate $\xi$ of these eigen modes corresponds to motion along diverging hyperbolic streamlines. However, the frequency $\nu$ is real which indicates linear stability of the flow with respect to weak fluctuations.

The corresponding eigen modes

$$
\begin{gathered}
\bar{\psi}_{1}(\xi)=-i \nu \frac{\bar{s}}{c \bar{\rho}} \frac{1+\alpha \xi}{\alpha \xi} \bar{\varphi}_{1}(\xi), \\
\bar{\psi}_{2}(\xi)=-i(\nu-k(\nu)) \frac{\bar{s}}{c \bar{\rho}} \bar{\varphi}_{2}(\xi)
\end{gathered}
$$

for the density fluctuations can be obtained directly from the linearization of the Euler equation (3).

Crossing the Mach horizon at $\xi=0$, the solution $\bar{\varphi}_{1}(\xi)$ acquires the factor $\exp \left\{\frac{\nu \pi}{\sqrt{3}}\right\}$. The same factor appears when considering quantum fluctuations. Following the logic of [2] (also discussed in detail in [21] - eqs. (2.88)-(2.90)) this results in radiation to the left of the Mach horizon (outside the "black hole") with the frequency distribution $N(\nu)=1 /\left(\exp \left\{\frac{\hbar \nu}{T_{H}}\right\}-1\right)$. This distribution has the same form as a Planck distribution for black body radiation, with the parameter $T_{H}=\frac{\sqrt{3} \bar{s} \hbar}{2 \pi c}$ in physical dimensions. The latter plays a role similar to that of the Hawking temperature in black hole radiation. However the quantity $T_{H}$ is not a real physical temperature. The part of time is played here by the spatial coordinate $z$, i.e. the "frequency" $\nu$ is the wave vector of a fluctuation in the $z$ direction. This "temperature" is measured in units of momentum rather than energy, and the corresponding "thermal fluctuations" are waves along the $z$ direction with a typical wavelength of order $\lambda_{H}=\hbar / T_{H}=2 \pi c /(\sqrt{3} s)$.

A "straddled" fluctuation

$$
\begin{gathered}
f(\xi, \zeta)=\int d \nu g(\nu) e^{-i \nu \zeta} \bar{\varphi}_{1}(\xi)= \\
\exp \left\{i\left[\nu_{0}+\sqrt{3} \Gamma^{2} \pi \theta(\xi)\right](\sqrt{3} \ln |\xi|+\zeta)\right\} \exp \left\{\left[\frac{\pi \nu_{0}}{\sqrt{3}}+\frac{\Gamma^{2} \pi^{2}}{6}\right] \theta(\xi)\right\} G(\xi, \zeta)
\end{gathered}
$$

$(\theta(\xi)$ is the step function) with a Gaussian spectral density $g(\nu)=\frac{1}{\Gamma \sqrt{2 \pi}} \exp \left\{-\frac{\left(\nu-\nu_{0}\right)^{2}}{2 \Gamma^{2}}\right\}$ around a positive frequency $\nu_{0}$ of a width $\Gamma$ is composed exclusively of the "left moving" normal modes $\bar{\varphi}_{1}(\xi)$, singular at $\xi=0$. Its "time" evolution (i.e. $\zeta$ dependence) is determined by the envelope function $G(\xi, \zeta)=$ $\exp \left\{-\frac{\Gamma^{2}}{2}(\sqrt{3} \ln |\xi|-\zeta)^{2}\right\}$. The "frequencies" of these oscillations on both sides of the horizon strongly vary with $\xi$. This is reminiscent of "time slowing" near the horizon of a real black hole.

Fig. 2] shows how the maxima of the fluctuation amplitude propagate on both sides of the Mach horizon, with zero amplitude on the horizon. The part residing to the right side of the horizon (the supersonic region, $\xi>0$ ) should be multiplied by the factor $\exp \left\{\frac{\pi \nu_{0}}{\sqrt{3}}+\frac{3 \Gamma^{2} \pi^{2}}{2}\right\}>$ 1 (not shown in Fig. 22), with the Hawking temperature $\pi \nu_{0} / \sqrt{3}=\hbar \nu_{0} / 2 T_{H}$ as a parameter. Therefore the ratio of amplitudes of a classical fluctuation on the left and the right of the horizon directly measures the Hawking temperature.

The fluctuation moves against the transonic flow with the velocity of sound. Its left part escapes to the left against the subsonic background flow, and moves with a very small velocity $s_{l}-v_{l} \approx \bar{s}-v_{l}$ to the left. The right part of the fluctuation is "flushed" away by the supersonic flow (see Fig. ??), and moves to the right with a small velocity $v_{r}-s_{r} \approx v_{r}-\bar{s}$. Here $v_{l}$ and $s$ are the subsonic flow velocity and local speed of sound of the flow in the vicinity and to the left of the Mach horizon, whereas $v_{r}$ and $s_{r}$ are the corresponding quantities in the supersonic region to the right of the Mach horizon (Note that for the symmetrical potential and spectral content of the fluctuation studied here the propagation on the two sides of the horizon is approximately symmetrical, as shown in Fig. (2). Using the eigen modes (.) for the density fluctuations we get a similar picture. In the case of quantum fluctuations the part moving to the left is analogous to the Hawking radiation observable outside the black hole. The part moving to the right is not observable in a real 


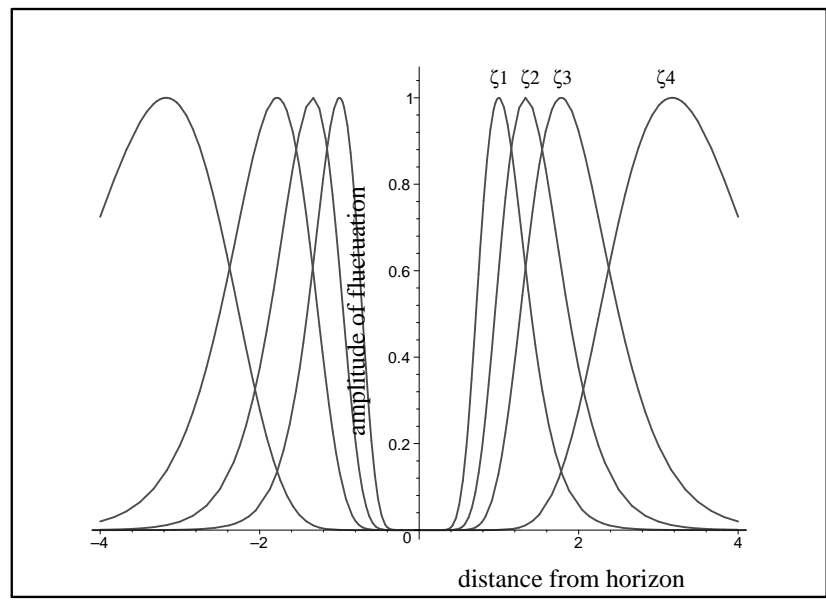

FIG. 2: Propagation of the straddled wave packet to the left and right of the Mach horizon. $\zeta_{1}=0, \zeta_{2}=0.5, \zeta_{3}=1$, $\zeta_{4}=2$ with $\Gamma=2$.

black hole. However in our case the supersonic region is accessible for observation.

The normal modes $\bar{\varphi}_{2}(\xi)$ in (10) are plane waves propagating to the right in the direction of the flow with the velocity $v_{2}=\bar{s}\left(\frac{d k(\nu)}{d \nu}\right)^{-1}$. This velocity is twice the sound velocity, i.e. the sound velocity on the background of the supersonic flow, for large enough "frequencies" $\nu \gg 1$. At small frequencies $\nu \ll 1$ the propagation velocity is closer to thrice the sound velocity, i.e. twice the sound velocity relative to the background flow. If we use the analogy with the real black holes this result would correspond to superluminal motion. However there is no paradox here since the wavelength is larger than the throat width in the Laval nozzle, $1 / k \gg c$ (in physical units). Hence we deal with the narrow field effect, analogous to superluminal propagation in near field optics and tunneling 222 24], which does not violate causality.

The above results are illustrated by numerical simulations of the NLS eq. (11) in $1+2$ dimensions using a finite-difference approach based on the split-step CrankNicholson method. 25] The potential $U(x, y)$ is zero outside the hyperbola (6) and a negative constant $U_{0}$ inside. A packet with a Gaussian density distribution is given a Galilean boost in the fashion similar to Ref. [26] with a velocity close to that of sound for the density in the maximum of the Gaussian. This configuration differs from the discussed in the analytical part of the paper and is chosen for its relative simplicity and due to the fact its experimental realization seems to be rather straightforward. Although it differs in many aspect such as strongly nonhomogeneous distribution of the density and, hence, of the sound velocity, which is also affected be the quantum potential contribution, this configuration may be very convenient and useful for studying propagation and classical fluctuations in the optical Laval nozzle.

The calculation is carried out for Eq. (11) with $\beta_{0}=5$ and $\lambda=10$, whereas the amplitude function $A$ is normalized to unity. The depth of the potential in the nozzle $U_{0}=-200$. It has a hyperbolic shape with $a=0.3$ and $b=7.5$. Meaning that its half-width is 0.3 at the throat at $x=0$ and 0.5 at $x= \pm 10$. The Gaussian centered initially at $x=-1, y=0$ is given a boost with the phase $\varphi=2.5 x$ corresponding to the unit velocity. Figs. 3 show the packet at four successive stages of its propagation. A straddled fluctuation gradually develops with a characteristic dip close to the throat of the nozzle, which becomes deeper and nearly cuts the original packet in two. The fluctuation in this case becomes strong and in the end consumes a major part of the packet.

Since the numerical code deals with the NLS eq. (1), it solves a nonlinear problem strongly deviating from the steady transonic flow assumed in the above analysis, and fully accounts for the quantum potential. The density is strongly nonhomogeneous which leads to a nonhomogeneous distribution of the sound velocity. The latter should be also corrected by the contribution of quantum potential which cannot be considered small anymore. The Gaussian packet evolves into a two hump structure permanently changing its shape with the propagation length $z$ ("time"), as is clearly demonstrated in Figs. 3,

There are such factors as quantum potential (dispersion) and defocusing nonlinearity, which may influence the dynamics of the packet and in principle also cause its splitting in two parts. Control calculations of the packet moving in free space, or in a potential well with parallel walls do not show the type of behavior shown in Fig. 3. Decreasing the boost velocity also weakens the effect. Otherwise the general pattern presented in these figures is rather robust and holds when varying the parameters as long as the packet is boosted in a hyperbolically shaped potential well.F

The above ideas can be tested experimentally by launching a continuous wave laser beam through an appropriately shaped nozzle, with reflective walls, filled with a Kerr-like defocusing nonlinear material. Such a one-dimensional nozzle can be constructed from two convex cylindrical mirrors, put back to back at an adjustable separation with the space between them sealed and filled with a nonlinear liquid such as iodine-doped ethanol [27]. The nonlinear coefficient $\lambda$ of the solution can be expressed, in terms of the nonlinearly-induced refractive index change $\delta n$, as $\delta n \beta_{0} / n_{0}$, where $n_{0}$ is the linear refractive index of the material [27]. The corresponding dimensionless sound velocity is $s=\sqrt{\delta n / n_{0}}$. Assuming a nonlinear index change of $1 \%$, an incoming laser beam would emulate supersonic flow when $k_{x} / \beta_{0}>0.1$, i.e. when the input angle of the beam is larger than $\approx 6$ degrees. The required input angle for observing transonic acceleration through the nozzle is therefore technically feasible. The acceleration can be controlled by mechanically varying the throat width, and monitored 

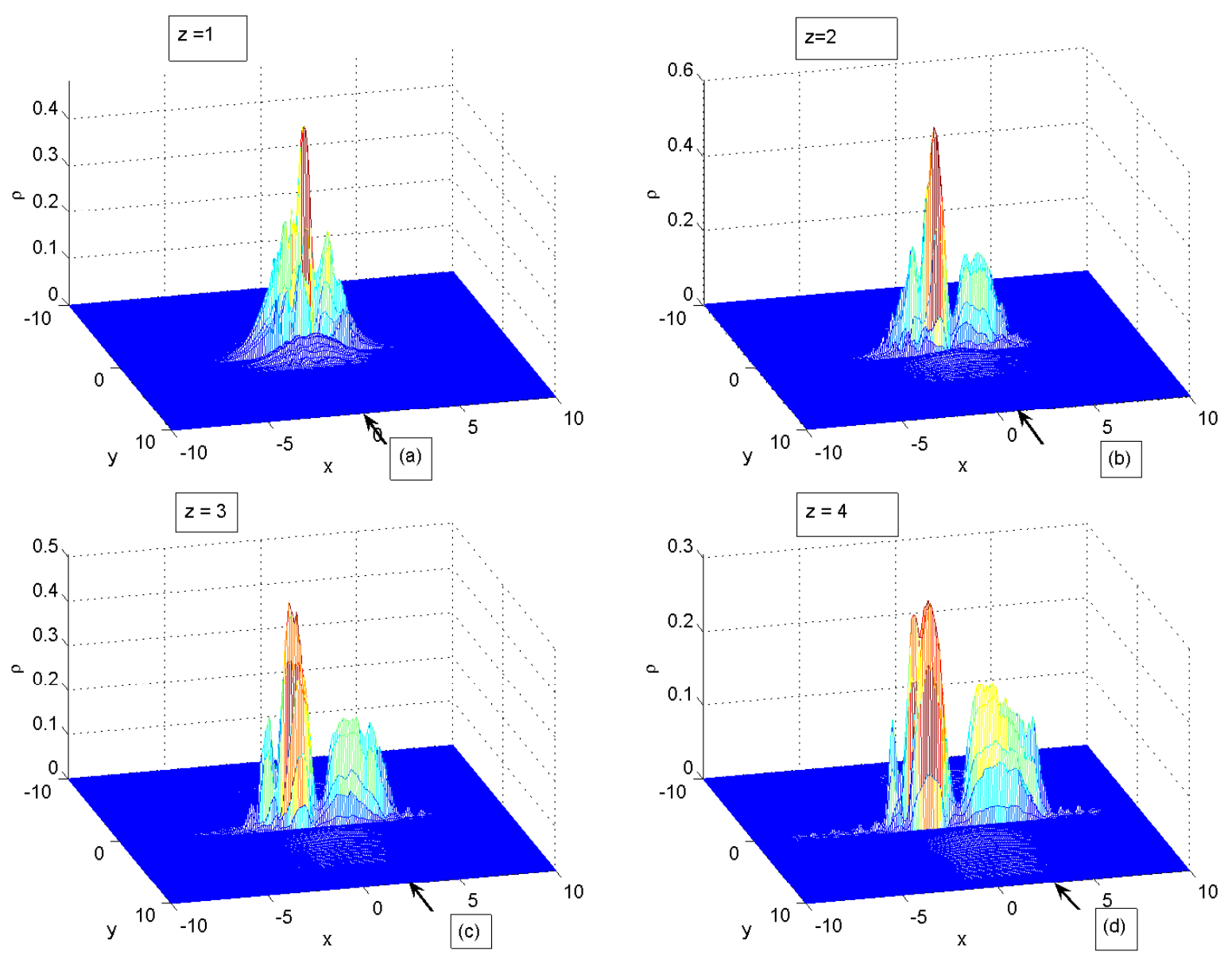

FIG. 3: (Color online) Four figures (a) to (b) show snapshots at different stages of the Gaussian packet motion through a hyperbolic Laval nozzle and development a straddled fluctuation with a characteristic dip close to the throat of the nozzle. The arrow in figure (a) to (d) shows the center of the packet if it were moving in a free space, which corresponds to the shift $\Delta x=1,2,3$ and 4, respectively, from the starting point $x=-1$. The propagation distance $z$ ("time") is indicated at each figure.

by observing the deflection of the laser beam as it traverses the nozzle. The intensity and phase profile of the beam coming out of the experimental setup can be studied using standard detection and interferometric techniques. These would allow measurement of the transverse acceleration of the beam, as well as detection and characterization of classical fluctuations that propagate away from the throat region. such classical fluctuations may be introduced by locally perturbing the optical fluid in a deterministic fashion, e.g. by injecting a second narrow laser beam into the nozzle throat 15 .

Constructing a Laval nozzle of hyperbolic shape might not be easy, but we believe that most of the effects discussed above will also be observed in other types of convergent-divergent nozzles (in fact many aeronautical applications of the Laval nozzle are not hyperbolically shaped). Otherwise the proposed experimental setup is quite simple and straightforward to implement in a tabletop experiment, in particular as compared to the previously proposed rotating black hole configuration [15] and to water tank experiments 28]. The (classical) fluctuations will be observed directly, superimposed on an otherwise smooth transverse beam profile, and in principle will not require as high a dynamic range as previous optical experiments [9]. The experiment is also more straightforward than other proposed schemes, e.g. those based on SQUID array transmission lines [8] and atomic BEC [6, 7].

In summary, we demonstrate the possibility of creating an optical analogue of the Laval nozzle. The interesting point will be to study the dynamics of straddled fluctuations which may be either quantum or classical and even artificially created. The equivalent of the Hawking temperature enters as an important parameter characterizing all types of fluctuations. This temperature is measured in units of momentum, rather than energy, which corresponds to a wavelength exceeding the width of nozzle throat by about two orders of magnitude, and in principle accessible for experimental measurements. Numerical simulations support the theoretical findings but also indicate that the phenomenon is much more complicated 
and interesting.

Acknowledgments. Support of Israeli Science Foundation, Grant N 944/05 and of United States - Israel Binational Science Foundation, Grant N 2006242 is acknowledged. VF is grateful to Max Planck Institute for Physics of Complex Systems, Dresden, for hospitality and to G. Shlyapnikov and N. Pavloff for stimulating discussions.

[1] Bekenstein J. D., Phys. Rev. D 7, 2333 (1973).

[2] Hawking S. W., Commun. Math. Phys. 43, 199 (1975).

[3] Unruh W. G., Phys. Rev. Lett. 46, 1351 (1981).

[4] Reznik B., Phys. Rev. D62, 044044 (2000).

[5] Barcelo C., Liberati S., Visser M., Phys. Rev. A 68, 053613 (2003).

[6] Carusotto I., Fagnocchi S., Recati A., Balbinot R. and Fabbri A., New Journal of Physics 10, 103001 (2008).

[7] Recati A., Pavloff N., and Carusotto I., Phys. Rev. A 80, 043603 (2009).

[8] Nation P. D., Blencowe M. P., Rimberg A. J., and Buks E., Phys. Rev. Lett. 103, 087004 (2009).

[9] Philbin T. G., Kuklewicz C., Robertson S., Hill S., König F., Leonhardt U., Science, 319, 1367 (2008).

[10] Lahav O., Itah A., Blumkin A., Gordon C., Steinhauer J., arXiv:0906.1337v1.

[11] Agrawal G. P., Nonlinear Fiber Optics, Aacademic Press, New York (1995).
[12] Madelung E., Z. Phys. 40, 322 (1927).

[13] Marburger J. H., Prog. Quant. Electr., 4, 35 (1975).

[14] Dekel G., Fleurov V., Soffer A. and Stucchio C., Phys. Rev. A 75, 043617 (2007).

[15] Marino F., Phys. Rev. A 78, 063804 (2008).

[16] Silberberg Y., Opt. Lett. 15, 1283 (1990).

[17] Sedov L. I., Two Dimensional Problems in Hydrodynamics and Aerodynamics, Interscience Publ., New York, 1965.

[18] Landau L. D. and Lifshits E. M., Fluid Mechanics, Pergamon Press, Oxford, 1987.

[19] Sakagami M. and Ohashi A., Prog. Theor. Phys. 107 (6), 1267 (2002).

[20] Barcelo C., Liberati S., and Visser M., Int. J. of Mod. Phys. A 18, 1 (2003).

[21] Damour T. and Lilley M., arXiv:0802.4169v1 [hep-th] (2008).

[22] Broe J. and Keller O., Journal of Microscopy, 202, 286 (2001).

[23] Mugnai D., Ranfagni A. and Ruggeri R., Phys. Rev. Lett. 84, 4830 (2000).

[24] Mugnai D., Ranfagni A. and Schulmann L.S., Phys. Lett. A 247, 291 (1998).

[25] Muruganandam P., Adhikari S.K., arXiv:0904.3131v [cond-mat.quant-gas] (2009).

[26] Frisch T., Pomeau Y., and Rica S., Phys. Rev. Lett. 69, 1644 (1992).

[27] Barsi C., Wan W., Sun C., and Fleischer J. W., Opt. Lett. 32, 2930 (2007).

[28] Rousseaux G., Mathis C., Maïssa P., Philbin T. G., and Leonhardt U., New Journal of Physics 10, 053015 (2008). 